\title{
Clinical and imaging characteristics of 53 ulcers of post-radiation nasopharyngeal necrosis in patients with nasopharyngeal carcinoma
}

\author{
FENGQIN YAN*, ZHIMIN YE*, FANGZHENG WANG, LEI WANG, WEIYANG LI and ZHENFU FU
}

Department of Radiation Oncology, Zhejiang Provincial Cancer Hospital, Hangzhou, Zhejiang 310022, P.R. China

Received March 18, 2016; Accepted June 23, 2016

DOI: $10.3892 / \mathrm{mco} .2016 .968$

\begin{abstract}
It is widely accepted that a mucosal ulcer induced by radiation (RIMU) is the predominant type of post-radiation nasopharyngeal ulcer in patients with nasopharyngeal carcinoma (NPC) who underwent radiotherapy (RT); however, another type of ulcer, an ulcer of post-radiation nasopharyngeal necrosis (UPRNN), has rarely been reported for patients with NPC. In the present study, the clinical and imaging features of 53 patients who were treated at the Zhejiang Provincial Cancer Center (Zhejiang Cancer Hospital, Hangzhou, China) between March 2009 and December 2015, and who were diagnosed with UPRNN, were reviewed. The clinical factors, laboratory examinations, magnetic resonance imaging (MRI) features and endoscopic findings were analysed. A UPRNN has its characteristic imaging features and ulcer locations at the primary tumour bed, which are different from a traditional RIMU. In the retrospective analysis of the clinical factors, a tumour (T) 3/4 stage, with invasion of muscular tissue, poor response of neoadjuvant chemotherapy and anaemia during the RT, may be associated with the occurrence of a UPRNN. To evaluate the severity, a UPRNN was divided into three grades according to the invasion depth of the ulcer based on its appearance in MRI, and the subsequent treatment and prognosis varied according to the severity of the UPRNN. In conclusion, a UPRNN has its clinical features and characteristic MRI appearances, and the occurrence of a UPRNN may be associated with several clinical factors.
\end{abstract}

\section{Introduction}

The mainstay of treatment for nasopharyngeal carcinoma (NPC) is radiotherapy (RT). Following the completion of RT,

Correspondence to: Dr Zhenfu Fu, Department of Radiation Oncology, Zhejiang Provincial Cancer Hospital, 38 Guangji Road, Hangzhou, Zhejiang 310022, P.R. China

E-mail: fuzf1000@163.com

\section{${ }^{*}$ Contributed equally}

Key words: nasopharyngeal carcinoma, radiation, necrosis, ulcer, magnetic resonance imaging a post-radiation nasopharyngeal ulcer (PRNU) is an important consequential adverse effect in patients with $\mathrm{NPC}(1,2)$. However, the diagnosis of PRNU has not been widely discussed, predominantly due to the lack of understanding and effective diagnosis for this complication. Traditionally, nasopharyngeal ulcers for patients who underwent RT are commonly divided into mucosal ulcers induced by radiation (RIMUs) and tumour necrosis-induced ulcers (TNIUs) $(3,4)$. The former belongs to a severe grade of radiation mucositis, which occurs in normal mucosa tissue; the latter is induced by the necrosis of tumour tissue, which usually accompanies tumour tissue.

In the present study, the intention was to elaborate on a third type of ulcer. This ulcer occurs following the collapse of radiation-induced tumour necrosis in areas of the primary tumour bed, which is the major characteristic differing from the two ulcers described above. This third type of ulcer has been termed 'ulcer of post-radiation nasopharyngeal necrosis' (UPRNN), which has been rarely reported to the best of our knowledge, although several authors have previously reported on certain NPC patients with post-radiation nasopharyngeal necrosis (PRNN) $(3,5)$. Only the simple phenomenon of PRNN was described in these studies, and the analysis of UPRNN with a full set of clinical characteristics, including occurrence, evolution, incentive factors, diagnosis, prognosis and grade of ulceration, has rarely been reported.

The present study aimed to illustrate the clinical characteristics and risk factors of UPRNN to better understand this complication.

\section{Patients and methods}

Patients. Between June 2010 and December 2015, 53 patients (13 women and 40 men) with pathologically diagnosed NPC were treated at the Department of Radiation Oncology, Zhejiang Provincial Cancer Hospital (Hangzhou, China). All these patients underwent intensity-modulated radiotherapy (IMRT), were diagnosed with a nasopharyngeal ulcer, and met the diagnosis criteria of a UPRNN. All the patients underwent a single round of IMRT, and all of them received chemotherapy, including induction chemotherapy for 34 patients, concurrent chemotherapy for 18 patients and adjuvant chemotherapy for 12 patients (Table I).

All the patients were followed up with MRI and endoscopic examinations every 3 months during the 2 years following RT, 
and subsequently 6 months after the 2 years. Once a nasopharyngeal ulcer had been diagnosed, pathological and MRI examinations were combined to identify the UPRNN. The follow-up period was defined from the completion of the RT to the last follow-up day.

MRI examination. All of the MRI examinations were performed with a 1.5-T MAGNETOM System (Siemens AG, Munich, Germany). The area from the suprasellar cistern to the inferior margin of the sternal end of the clavicle was scanned with a head-and-neck combined coil. The scanning sequence included transverse ( $\mathrm{T}$ ) 1-weighted images on the axial, coronal and sagittal planes, and the T2-weighted images on the axial plane were obtained prior to the injection of a contrast reagent. Following an intravenous gadolinium-diethylenetriamine pentaacetic acid (Gd-DTPA) injection at a dose of $0.1 \mathrm{mmol} / \mathrm{kg}$ of body weight, T1-weighted axial and sagittal sequences were performed sequentially. The MRI examinations were interpreted by a radiologist and a radiation oncologist experienced in NPC diagnoses and treatment for 10 years, respectively.

Diagnosis and grading of UPRNN. The diagnosis criteria of a UPRNN were established in order to conform with the following points: i) The pathological confirmation included typical features of an ulcer without evidence of tumour recurrence or any residual tumour; ii) the MRI examination revealed an ulcer site at the primary tumour bed; and iii) the patients had previously undergone RT. The grading of the UPRNN was divided into three degrees according to the depth of the ulcer on MRI examination: A mild-grade UPRNN was classified as an ulcer confined to the mucosa (Fig. 1); a moderate-grade UPRNN was an ulcer invading, but not exceeding, the muscle tissue (Fig. 2); and a severe-grade UPRNN was an ulcer exceeding the muscle tissue (Fig. 3).

Laboratory examination. All the patients accepted a routine laboratory examination every week during the course of RT, including routine blood and biochemical testing. All the above examinations were reviewed from the medical record.

Treatment of UPRNN. According to the severity of the UPRNN, the patients with a mild or moderate grade UPRNN were administered a conservative treatment, including anti-inflammation treatment and flushing with $0.9 \%$ saline. The patients with a severe UPRNN were treated with endoscopic debridement and anti-inflammation treatment.

Statistical analyses. All statistical analyses were performed using SPSS 16.0 software (SPSS, Inc., Chicago, IL, USA). Descriptive statistics were produced for continuous variables. The results are presented as the mean \pm SD for continuous variables.

\section{Results}

General clinical feature of UPRNN. The general clinical data of the 53 patients included in the present study are summarised in Table I. All the 53 patients complained of headache and experienced a foul odour. The average age was
$46.75 \pm 6.82$ years. The body mass index was $23.47 \pm 2.67 \mathrm{~kg} / \mathrm{m}^{2}$, and the prescriptive dose of the ulcer zone was $70.59 \pm 2.36 \mathrm{~Gy}$. As for the tumour (T) stage, the UPRNN was predominantly distributed at the T3/4 stage. All the patients underwent chemotherapy, including neo-adjuvant chemotherapy for 34 patients, concurrent chemotherapy for 18 patients and adjuvant chemotherapy for 12 patients. In the analysis of the neo-adjuvant chemotherapy response, 22 patients were categorised as having stable disease (SD), and 12 patients received a partial response. According to the depth of invasion, the UPRNN was divided into three grades, including four mild-grade UPRNNs, 27 moderate-grade UPRNNs and 22 severe-grade UPRNNs. The average interval from the completion of RT to the initiation of UPRNN was $2.92 \pm 1.26$ months.

In the laboratory examinations, the results revealed that 47 patients also had anaemia during the course of RT, which included 27 patients with mild anaemia, 20 with moderate anaemia and none with severe anaemia. However, as for an infectious marker, the majority of the patients were diagnosed with a normal value of C-response protein during the course of RT (Table II). During the follow-up period, 48 patients were alive, whereas five patients succumbed to mortality due to a massive nasopharyngeal haemorrhage. In the analysis of the sites that contained an ulcer, the parts especially for medial/lateral pterygoid muscle, and musculus longus capitis involved by tumour, was easier to complicate with UPRNN (Table III).

MRI and endoscopic features of patients with UPRNN. The results of the MRI examinations, combined with the endoscopic examinations, were revealed to be well consistent with UPRNN. It is a good modality for an MRI examination to estimate the invasion scope of a moderate- or severe-grade UPRNN, whereas it is difficult to detect a mild-grade UPRNN from an MRI examination alone. From an MRI examination, UPRNNs are revealed to commonly present with different sizes of defects located in the primary tumour bed, unless there is an adhesion of necrosis or secreta. However, it may be advantageous for a diagnosis of UPRNN to be made using contrast-enhanced MRI, which highlights non-enhanced zones in the UPRNN.

Treatment and prognosis of UPRNN. Of the four patients with a mild-grade UPRNN, three of them were stable and ultimately recovered following an anti-inflammation and flushing treatment, although the condition of one patient deteriorated into moderate-grade UPRNN, and this patient underwent conservative treatment. None of the patients succumbed to mortality. Of the 27 patients with a moderate-grade UPRNN, 14 were stable and finally recovered, although 13 patients were progressive and underwent debridement. One of them succumbed to mortality. Of the 22 patients with a severe-grade UPRNN, none of them were stable and all the patients underwent endoscopic debridement. Four of the patients succumbed to mortality due to a massive haemorrhage (Table IV).

\section{Discussion}

RT, as the most effective treatment modality, exerts a vital role in NPC treatment, while it is a kopis for the treatment as increasing numbers of patients are experiencing various 
Table I. Characteristics of 53 patients with nasopharyngeal carcinoma who had post-radiation nasopharyngeal necrosis.

\begin{tabular}{|c|c|}
\hline Characteristic & UPRNN \\
\hline \multicolumn{2}{|l|}{ Age (years) } \\
\hline Mean \pm SD & $46.75 \pm 6.82$ \\
\hline \multicolumn{2}{|l|}{ Gender } \\
\hline Male & 32 \\
\hline Female & 21 \\
\hline \multicolumn{2}{|l|}{ BMI } \\
\hline Mean \pm SD & $23.47 \pm 2.67$ \\
\hline \multicolumn{2}{|c|}{ Prescriptive dose of ulcer zone (Gy) } \\
\hline Mean \pm SD & $70.59 \pm 2.36$ \\
\hline \multicolumn{2}{|l|}{ T stage } \\
\hline $\mathrm{T} 1$ & 0 \\
\hline $\mathrm{T} 2$ & 2 \\
\hline T3 & 36 \\
\hline $\mathrm{T} 4$ & 15 \\
\hline \multicolumn{2}{|l|}{ Sites of involvement ${ }^{b}$} \\
\hline $1-3$ & 24 \\
\hline$>3$ & 29 \\
\hline \multicolumn{2}{|l|}{ Grades of ulcer } \\
\hline Mild & 4 \\
\hline Moderate & 27 \\
\hline Severe & 22 \\
\hline \multicolumn{2}{|l|}{ Chemotherapy } \\
\hline Neo-CT & 34 \\
\hline Concurrent & 18 \\
\hline Adjuvant & 12 \\
\hline \multicolumn{2}{|l|}{ Response of neo-CT } \\
\hline $\mathrm{SD}$ & 22 \\
\hline PR & 12 \\
\hline CR & 0 \\
\hline \multicolumn{2}{|l|}{ Interval $^{\mathrm{a}}$ (months) } \\
\hline Mean \pm SD & $2.92 \pm 1.26$ \\
\hline \multicolumn{2}{|l|}{ Outcome of UPRNN } \\
\hline Alive & 48 \\
\hline Succumbed to mortality & 5 \\
\hline
\end{tabular}

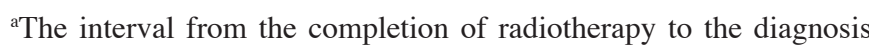
of ulcer of post-radiation nasopharyngeal necrosis. ${ }^{\text {b}}$ The sites of involvement included the medial pterygoid muscle, lateral pterygoid muscle, parapharyngeal space, musculus longus capitis and the skull base. BMI, body mass index; neo-CT, neoadjuvant chemotherapy; $\mathrm{SD}$, stable disease; $\mathrm{PR}$, partial response; $\mathrm{CR}$, complete response; $\mathrm{SD}$, standard deviation; UPRNN, ulcer of post-radiation nasopharyngeal necrosis.

post-radiation complications, corresponding to the increasing number of survivors of irradiated NPC. An RIMU in NPC, as a severe grade of post-radiation nasopharyngeal mucositis for patients with NPC, was considered to be the only type of ulcer other than the tumorigenic ulcer (6). However, the present hypothesis is that a third type of ulcer that is different from an
Table II. Laboratory examination of 53 patients with UPRNN.

\begin{tabular}{lc}
\hline Item & No. of patients \\
\hline Blood routine & 6 \\
Normal & 27 \\
Mild anaemia $^{\mathrm{a}}$ & 20 \\
Moderate anaemia & 0 \\
Severe anaemia & \\
C-response protein $^{\text {Normal }}$ & 31 \\
2 folds of normal & 18 \\
10 folds of normal $^{\text {b }}$ & 4 \\
\hline
\end{tabular}

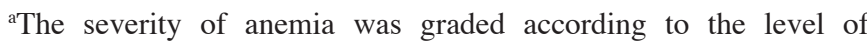
hemoglobin. ${ }^{\mathrm{b}}$ The normal level of C-response protein was $0-10 \mathrm{mg} / \mathrm{l}$.

Table III. Involvement of parts of the tumour in 53 patients with UPRNN.

\begin{tabular}{lc}
\hline Part & No. of patients \\
\hline Medial pterygoid muscle & 36 \\
Lateral pterygoid muscle & 15 \\
Parapharyngeal space & 53 \\
Musculus longus capitis & 43 \\
Skull base & 12
\end{tabular}

UPRNN, ulcer of post-radiation nasopharyngeal necrosis.

Table IV. Distribution and prognosis of the 53 patients with UPRNN.

Grade of

UPRNN Stable Debridement Progressive Dead

\begin{tabular}{lrrrr}
\hline Mild & 3 & 0 & 1 & 0 \\
Moderate & 14 & 13 & 13 & 1 \\
Severe & 0 & 22 & 22 & 4
\end{tabular}

UPRNN, ulcer of post-radiation nasopharyngeal necrosis.

RIMU may exist when a patient undergoes RT, which in the present study has been termed a 'UPRNN'.

In the present study, 53 patients were included who met our diagnosis criteria for a UPRNN, and the clinical characteristics of a UPRNN were analysed. Based on the results obtained, three clinical factors were identified that may be associated with the occurrence of a UPRNN. First, the occurrence of nasopharyngeal necrosis is associated with the response to neoadjuvant chemotherapy for NPC in the study. The results demonstrated that 22 of the 34 patients who underwent neo-chemotherapy were of SD and suffered from a UPRNN, which revealed that those patients that respond poorly to 

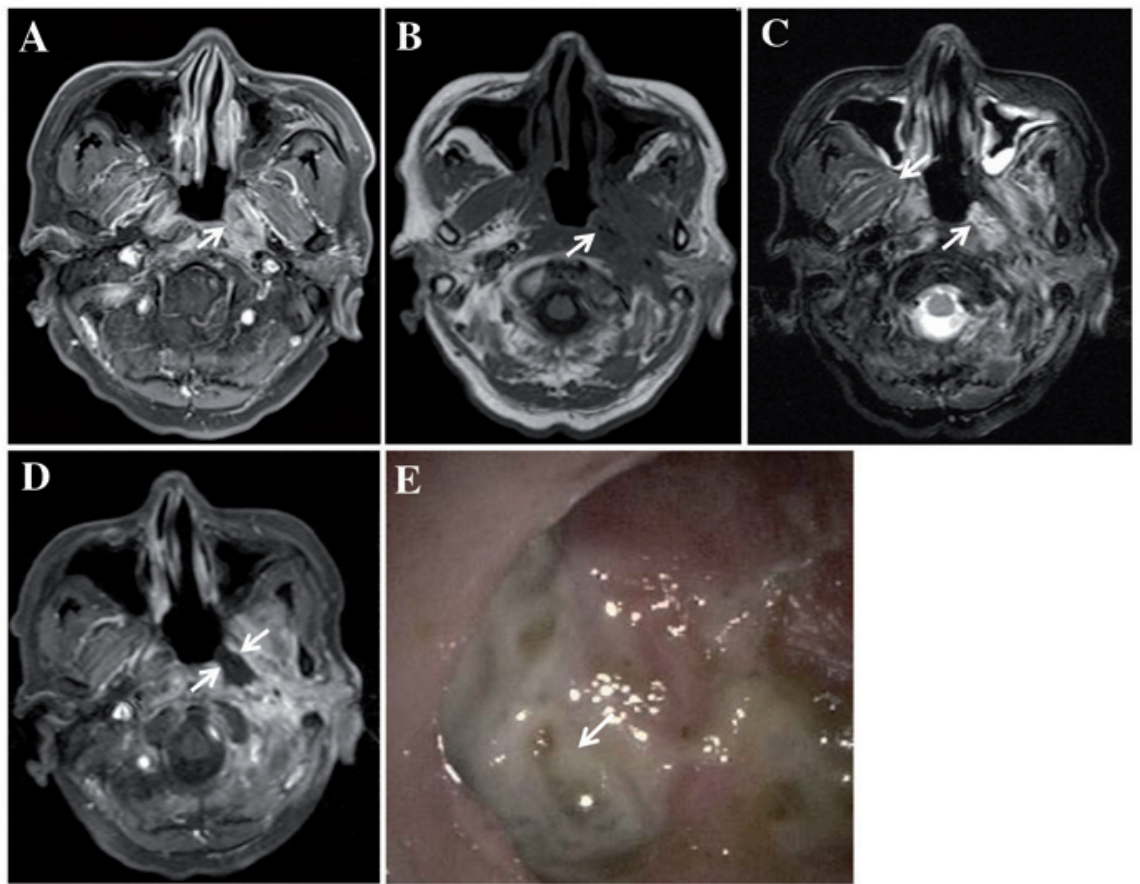

Figure 1. MRI examination performed on a 63-year-old man diagnosed with NPC with T3N2M0, who underwent IMRT without neo-adjuvant chemotherapy. (A) Transverse contrast-enhanced, T1-weighted MRI revealed enhanced tumour tissue invading the musculus longus capitis and medial pterygoid muscle on the left side of the nasopharyngeal cavity. (B) Transverse T1-weighted MRI revealed a low-signal area in the initial tumour bed at 2 months following the completion of RT (as indicated by the arrow). (C) Transverse T2-weighted MRI revealed a high-signal area in the initial tumour bed (indicated by the arrow). (D) Transverse contrast-enhanced, T1-weighted MRI revealed a non-enhanced area, which showed the ulcer exceeding the muscle tissue (arrow), and this was categorised as a severe-grade UPRNN. (E) The ulcer was located on the left side of the nasopharyngeal cavity (demonstrated by the arrow). RT, radiotherapy; MRI, magnetic resonance imaging; NPC, nasopharyngeal carcinoma; IMRT, intensity-modulated RT; UPRNN, ulcer of post-radiation nasopharyngeal necrosis.
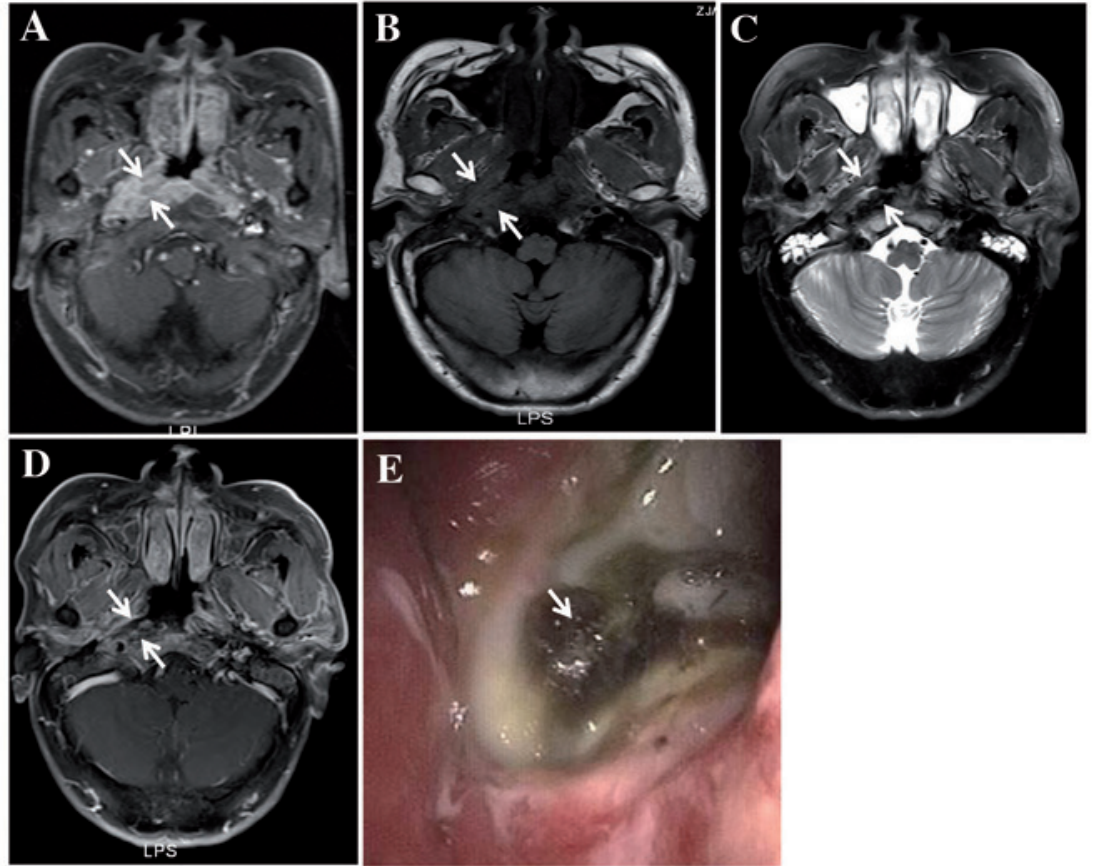

Figure 2. MRI examination performed on a 64-year-old man diagnosed with NPC with T3N3M0, who underwent IMRT with two courses of neo-adjuvant chemotherapy and received a partial response of short-term therapeutic effects of chemotherapy. (A) Transverse contrast-enhanced T1-weighted MRI revealed enhanced tumour tissue invading the musculus longus capitis, parapharyngeal space and medial pterygoid muscle on the right side of the nasopharyngeal cavity (as indicated by the arrows). (B) Transverse T1-weighted MRI revealed a low-signal area in the initial tumour bed at 1 month following the completion of RT (demonstrated by the arrows). (C) Transverse T2-weighted MRI revealed a mixed signal area in the initial tumour bed (shown by the arrows). (D) Transverse contrast-enhanced, T1-weighted MRI revealed a non-enhanced area, which showed the ulcer invading, but not exceeding, the muscle tissue (demonstrated by the arrows). The ulcer was categorised as a moderate-grade UPRNN. (E) The ulcer was located on the right side of the nasopharyngeal cavity (indicated by the arrow). RT, radiotherapy; MRI, magnetic resonance imaging; NPC, nasopharyngeal carcinoma; IMRT, intensity-modulated RT; UPRNN, ulcer of post-radiation nasopharyngeal necrosis. 

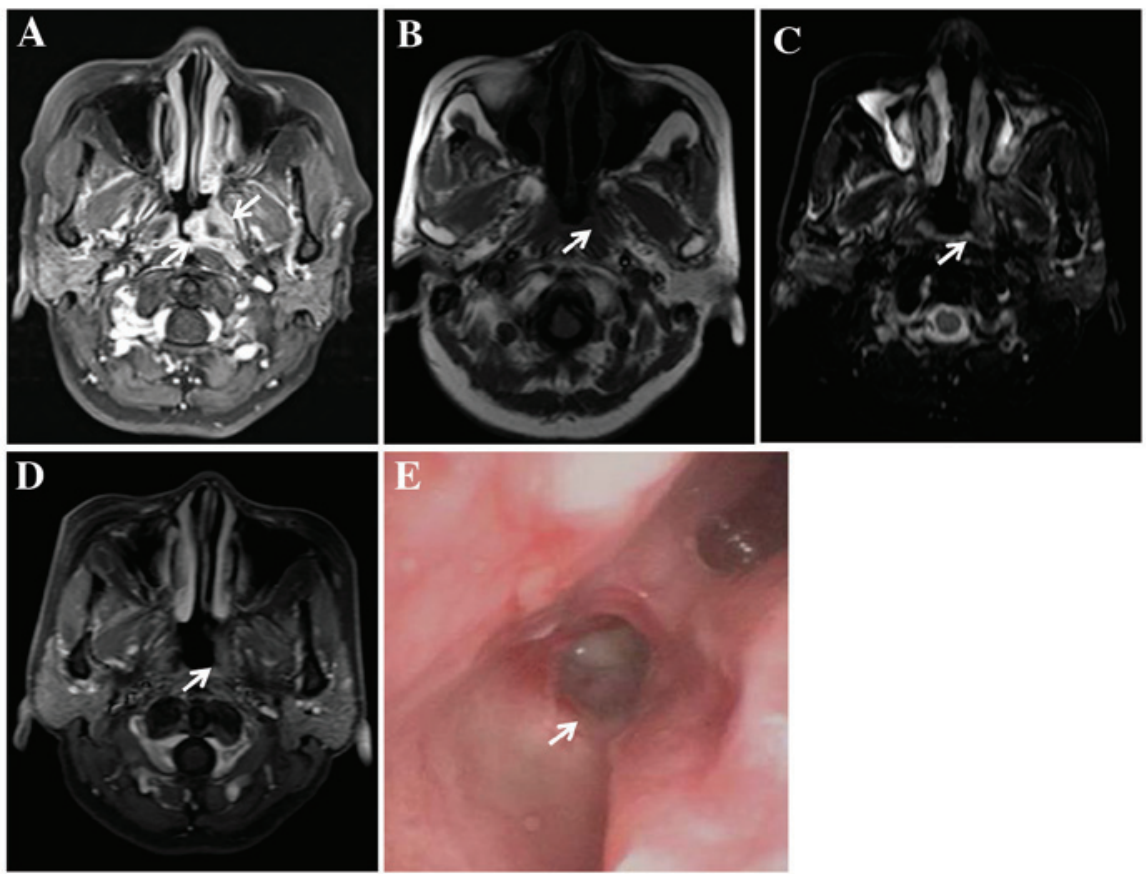

Figure 3. MRI examination performed on a 53-year-old woman diagnosed with NPC with T3N1M0, who underwent IMRT without neo-adjuvant chemotherapy. (A) Transverse contrast-enhanced, T1-weighted MRI revealed enhanced tumour tissue invading the parapharyngeal space and medial pterygoid muscle on the left side of the nasopharyngeal cavity (shown by the arrows). (B) Transverse T1-weighted MRI revealed a small defect in the nasopharyngeal mucosa of the initial tumour bed just after the completion of RT (shown by the arrow). (C) Transverse T2-weighted MRI also revealed a small defect in the nasopharyngeal mucosa of the initial tumour bed (shown by the arrow). (D) Transverse contrast-enhanced T1-weighted MRI revealed a non-enhanced area, which showed the ulcer limited in the mucosa tissue (indicated by the arrow). The ulcer was categorised as a mild-grade UPRNN. (E) The ulcer was located on the left side of the nasopharyngeal cavity (indicated by the arrow)). RT, radiotherapy; MRI, magnetic resonance imaging; NPC, nasopharyngeal carcinoma; IMRT, intensity-modulated RT; UPRNN, ulcer of post-radiation nasopharyngeal necrosis.
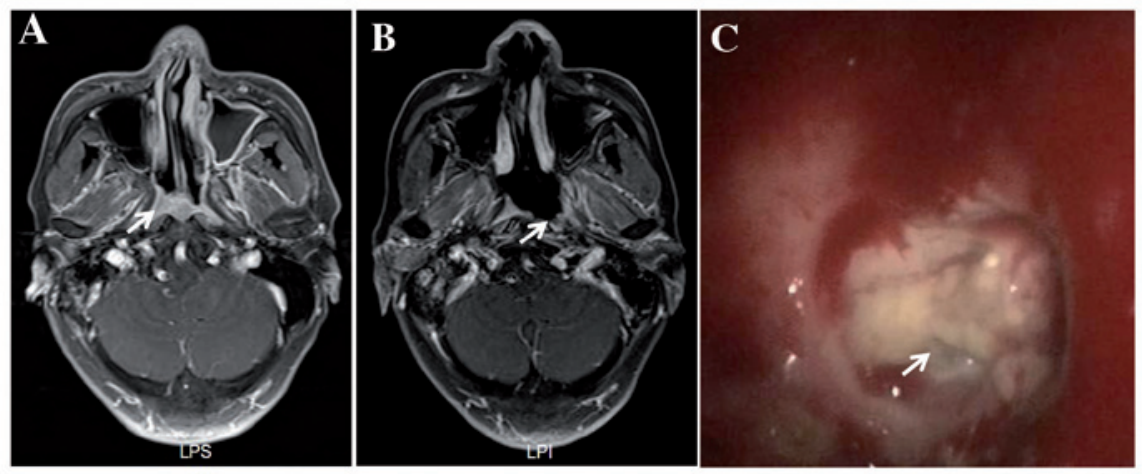

Figure 4. MRI examination performed on a 69-year-old man diagnosed with NPC with T3N2M0/rT3N0M0, who underwent RT twice. The second RT was IMRT with two courses of neo-adjuvant chemotherapy. The ulcer occurred at 4 months following the completion of IMRT. The patient was diagnosed with RIMU, not UPRNN. (A) Transverse contrast-enhanced, T1-weighted MRI revealed enhanced tumour tissue invading the parapharyngeal space and medial pterygoid muscle on the left side of the nasopharyngeal cavity (shown by the arrow). (B) Transverse contrast-enhanced, T1-weighted MRI revealed a large ulcer with non-enhancement on the right side of the nasopharyngeal cavity at 4 months following the completion of RT, which was not previously occupied by clear tumour tissue (shown by the arrow). (C) The ulcer was located on the left side of the nasopharyngeal cavity (indicated by the arrow). RT, radiotherapy; MRI, magnetic resonance imaging; NPC, nasopharyngeal carcinoma; IMRT, intensity-modulated RT; RIMU, mucosal ulcer induced by radiation; UPRNN, ulcer of post-radiation nasopharyngeal necrosis.

treatment may be more prone to suffering from a UPRNN. Secondly, the present results revealed that the majority of the patients with UPRNN also had anaemia, which demonstrated that malnutrition may exert an important role in the development of a UPRNN. Thirdly, it remained the case that the lesion area and $\mathrm{T}$ stage were more closely associated with the occurrence of UPRNN. The invasion of muscle (particularly for the musculus longus capitis and medial/lateral pterygoid muscle) and T3/4 stage have a tendency to complicate a UPRNN. On the other hand, inflammatory factors did not appear to be associated with the occurrence of a UPRNN.

The exact mechanism underlying the formation of a UPRNN has yet to be fully elucidated. It has been reported that hypoxia, hypovascularity and hypocellularity may be caused by RT in areas occupied by a tumour. Consequently, normal collagen synthesis and cell production may be impaired due 
to malnutrition and a deficiency of oxygen, which may subsequently lead to tissue breakdown and a chronic, non-healing wound (7). In the present study, factors, including poor response of chemotherapy, anaemia and large bulk of a tumour, seem to be in accordance with the above hypothesis. A large tumour, and poor response of a tumour, are predictive of a deficiency of the blood supply and oxygen in the parenchyma of a tumour. Additionally, the complication of anaemia during the course of RT demonstrated that malnutrition may exert an important role in the development of UPRNN.

An RIMU in NPC, a major severe late adverse effect, was reported with an incidence as high as 31.5-40.6\%. It is one of the complications attributed to RT-induced mucositis $(1,5)$, and should be distinguished from a UPRNN. To the best of our knowledge, the location of an RIMU usually originates from normal mucosa tissue, and the onset time of an RIMU is 1 year following the completion of RT (Fig. 4). However, the site of a UPRNN is closely associated with the site of the primary tumour, and the onset time of a UPRNN is usually within 6 months following the completion of RT. A UPRNN may be cured in that early period (Figs. 1-3). It may be helpful to differentiate both types of ulcer via MRI and endoscopic examinations. In the present study, the UPRNNs were ranked into three grades according to the depth of invasion, and it was revealed that a severe-grade UPRNN should be treated with debridement. Otherwise, the UPRNN may worsen and endanger the life of the patient.

The MRI characteristics of a UPRNN usually reveal defects in the region of the primary tumour bed, unless there is an adhesion of the pseudomembrane or necrosis, and a non-enhanced region is observed in areas of UPRNN. A mild-grade UPRNN exhibits discontinuous enhanced mucosa, which can be easily overlooked due to the small size of the ulcer. An endoscopic examination is helpful in diagnosing a mild-grade UPRNN. An MRI examination allows for the assessment of the scope of the UPRNN; therefore, the UPRNNs were divided into three grades according to the invasion depth.

The treatment of a UPRNN is diverse, according to the severity of the UPRNN. A mild-grade UPRNN may call for a conservative treatment or a follow-up examination, and a moderate-grade UPRNN should be treated medically, with a subsequent follow-up examination. If the disease is progressive, debridement may be necessary for the patient. A patient with a severe-grade UPRNN should be given endoscopic debridement and irrigation of the nasal cavity. The prognosis of a UPRNN also differs from the severity of the UPRNN. For example, a patient with a mild/moderate-grade UPRNN may recover from the disease after medical treatment has been administered; however, a severe-grade UPRNN may be fatal unless debridement is given.
As for the pathological development of a UPRNN, this may be described as a process of three stages through dynamic observation. In the early stage, necrosis occurs in the region of the tumour bed. In the second stage, granulation tissue may be observed around the area of necrosis, and in the last stage, scar tissue may be formed in the region of the granulation tissue. However, the above three stages are not observed in all the patients, since the majority of the patients are not followed up at regular intervals or do not visit the hospital in time.

In conclusion, in the present study UPRNNs have been demonstrated to possess characteristic clinical features and a characteristic appearance following MRI, and the occurrence of a UPRNN may be associated with several clinical factors. Therefore, the recognition of UPRNNs may be useful as a prerequisite in terms of their treatment and avoidance.

\section{Acknowledgements}

The present study was supported by grants from Zhejiang Provincial Medical Science and Technology Program (grant no. 2015RCB005), the Provincial Natural Science Funds of the Zhejiang Province of China (grant no. Q15H160012), and the General Project of the Zhejiang Provincial Health Bureau (grant nos. 2015KYB063, 2015KYB048 and 2016KYA051).

\section{References}

1. Qiu S, Lin S, Tham IW, Pan J, Lu J and Lu JJ: Intensity-modulated radiation therapy in the salvage of locally recurrent nasopharyngeal carcinoma. Int J Radiat Oncol Biol Phys 83: 676-683, 2012 .

2. Wu X, Huang PY, Peng PJ, Lu LX, Han F, Wu SX, Hou X, Zhao HY, Huang Y, Fang WF, et al: Long-term follow-up of a phase III study comparing radiotherapy with or without weekly oxaliplatin for locoregionally advanced nasopharyngeal carcinoma. Ann Oncol 24: 2131-2136, 2013.

3. Chen MY, Mai HQ, Sun R, Guo X, Zhao C, Hong MH and Hua YJ: Clinical findings and imaging features of 67 nasopharyngeal carcinoma patients with postradiation nasopharyngeal necrosis. Chin J Cancer 32: 533-538, 2013.

4. Hua YJ, Chen MY, Qian CN, Hong MH, Zhao C, Guo L, Guo X and Cao KJ: Postradiation nasopharyngeal necrosis in the patients with nasopharyngeal carcinoma. Head Neck 31: 807-812, 2009.

5. Chen HY, Ma XM, Ye M, Hou YL, Xie HY and Bai YR: Effectiveness and toxicities of intensity-modulated radiotherapy for patients with locally recurrent nasopharyngeal carcinoma. PLoS One 8: e73918, 2013.

6. Kwong DL, Nicholls J, Wei WI, Chua DT, Sham JS, Yuen PW, Cheng AC, Yau CC, Kwong PW and Choy DT: Correlation of endoscopic and histologic findings before and after treatment for nasopharyngeal carcinoma. Head Neck 23: 34-41, 2001.

7. Marx RE: Osteoradionecrosis: A new concept of its pathophysiology. J Oral Maxillofac Surg 41: 283-288, 1983. 